\title{
Intermittent Flow In Yield-Stress Fluids Slows Down Chaotic Mixing
}

\author{
D. M. Wendell, F. Pigeonneau, E. Gouillart, and P. Jop \\ Surface du Verre et Interfaces, UMR 125 CNRS/Saint-Gobain, \\ 39, quai Lucien Lefranc, F-93303 Aubervilliers, Cedex, France
}

(Dated: May 21, 2022)

\begin{abstract}
In this article, we present experimental results of chaotic mixing of Newtonian fluids and yield stress fluids using rod-stirring protocol with rotating vessel. We show how the mixing of yield stress fluids by chaotic advection is reduced compared to the mixing of Newtonian fluids and explain our results bringing to light the relevant mechanisms: the presence of fluid that only flows intermittently, a phenomenon enhanced by the yield stress, and the importance of the peripheral region. This finding is confirmed via numerical simulations. Anomalously slow mixing is observed when the synchronization of different stirring elements leads to the repetition of slow stretching for the same fluid particles.

PACS numbers: $47.51 .+\mathrm{a} 47.52 .+\mathrm{j} 47.50 .-\mathrm{d}$
\end{abstract}

Many mixing situations involve fluids with nonNewtonian properties: mixing of building materials such as concrete or mortar are based on fluids that have shearthinning rheological properties. Lack of correct mixing can waste time and money, or lead to products with defects [1. When fluids are stirred and mixed together at low Reynolds number, the fluid particles should undergo chaotic trajectories to be well mixed by the so-called chaotic advection [2] resulting from the flow. Previous work to characterize chaotic mixing in many different geometries has primarily focused on Newtonian fluids [3]. First studies into non-Newtonian chaotic advection often utilize idealized mixing geometries such as cavity flows or journal bearing flows for numerical studies [4] 6 .

In this article, we present results of mixing of Newtonian fluids and yield-stress fluids. We implement a mixing protocol inspired by the figure-eight stirring with rotating vessel described in [7. We observe the effects of the yield stress in the characteristic shape of the mixing area and in the mixing rate when varying the absolute velocity of the system. We show that the ratio of rotational velocity between the stirring rods and the wall of the vessel significantly influences the long-time mixing behavior of this mixing protocol. Resonances at integer values of this ratio are shown to lead to slow mixing. $\mathrm{Nu}-$ merical simulations are utilized to calculate the average area below the yield stress. We find that areas of slow or intermittent stretching due to the rheological properties of the fluid slow down mixing of the whole system.

We begin with a description of the apparatus. All experiments investigate 2D flows with four periodicallydriven cylindrical stirring rods rotating with constant angular velocity in an eggbeater configuration (Fig. 1a), designed to promote chaotic advection, inside of a cylindrical mixing vessel with a diameter of $14 \mathrm{~cm}$. The mixing vessel can also be rotated at constant angular velocity. The importance of the vessel rotation has been discussed in [7 9]. The experimental parameters can be found in Table I. The experiments are characterized by the stir-
TABLE I. Mixing Rate Experimental Parameters. The stirring ratio, $S$, is the mixing vessel rotation period divided by the stirring rod rotation period.

\begin{tabular}{ccc}
\hline \hline$S$ Ratio & Vessel Period [sec] & Rod Period [sec] \\
4.00 & 24 & 6 \\
4.33 & 26 & 6 \\
10.33 slow & 62 & 6 \\
10.33 fast & 31 & 3 \\
25.83 slow & 155 & 6 \\
\hline \hline
\end{tabular}

ring ratio, $S$, the ratio between the period of rotation of the vessel and the period of rotation of the stirring rods. Three fluids were used in the experiments: cane sugar syrup, a Newtonian fluid with a kinematic viscosity of $5 \times 10^{-4} \mathrm{~m}^{2} \mathrm{~s}^{-1}$, and 0.1 w.t.\% and 0.2 w.t\% Carbopol polymer solutions from Lubrizol, which are yield stress (YS) fluids characterized by the Herschel-Bulkley law [10. A spot of low-diffusivity dye (Colorex trichromatic black, from Pebeo) is injected in the middle depth of the vessel to minimize surface effects. We follow the evolution of the dye concentration field during the mixing process by taking photographs through the transparent bottom of the mixing vessel once per period of the rods. In addition, numerical simulations are realized with a finite-element method. The constitutive equation of Herschel-Bulkley law is regularized according the model of Deglo de Besses et al. [11. The area where the fluid is in solid rotation is extracted along one period of rotation of the rods.

The topology of the mixing region has several interesting features. Representative photographs are shown in Fig. 1 b-h. The fluid domain is separated into a large chaotic region delineated by the long-term dye pattern, and a non-chaotic dye-free region where particles are entrained on closed trajectories by the rotating wall [7]. For the yield-stress fluid, the width of the non-chaotic region is larger than for the Newtonian fluid because of a zone of intermittent solid rotation close to the rotating 

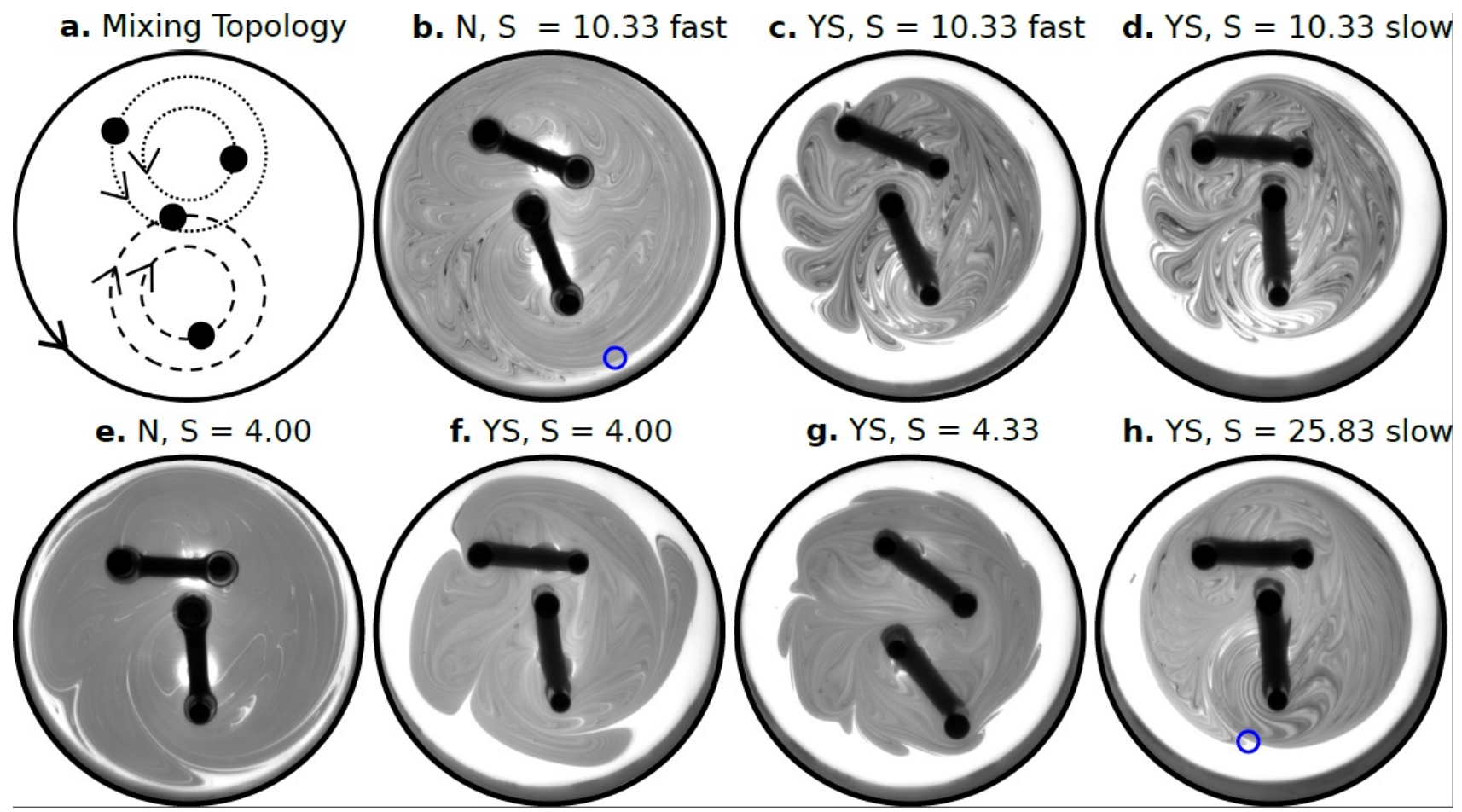

FIG. 1. (Color online) The mixing protocol and selected results. All times, $t$, are normalized by the period of the stirring rods. The fluid composition is indicated by $\mathrm{N}$ for Newtonian fluid and YS for yield stress fluid. [a] The stirring protocol. Note the four rotating stirring rods and the direction of rotation of the vessel. [b] Newtonian fluid, $S=10.33$, fast velocity, $t=20$. [c] 0.1 w.t.\% YS fluid, $S=10.33$, fast velocity, $t=20$. [d] 0.1 w.t.\% YS fluid, $S=10.33$, slow velocity, $t=20$. [e] Newtonian fluid, $S=4.00, t=50$. [f] 0.1 w.t. $\%$ YS fluid, $S=4.00, t=50$. [g] 0.1 w.t.\% YS fluid, $S=4.33, t=50$. [h] 0.1 w.t. $\%$ YS fluid, $S=25.83$, $t=40$. The tiny blue circles represent fixed (1-periodic) points.

vessel. Its boundary corresponds roughly to the largest extent of the rods' trajectories. For a slow velocity of the vessel, the mixing pattern displays a single injection point (blue circle in Fig. 1 1 and 1 $\mathrm{h}$ ). The stroboscopic position of this point is fixed at every period of the rods, and stems from the competition between the moving wall and the lower pair of rods. When the speed of the wall increases, this fixed (periodic) point is shifted within the chaotic region. Then, fluid particles inside the chaotic region but close to its boundary circle along the rotating wall for a few periods, before escaping to the center of the mixing region. In this case, the border of the chaotic region shows several lobes, that are almost-cyclic sets [12] delineated by dye-free cusps [13] (Fig. 1k-g). For the yield-stress fluid, fluid inside the lobes is only intermittently sheared, due to the proximity of the wall. The number of lobes is approximately equal to the stirring ratio. The transition from one fixed point to travelling lobes occurs earlier when increasing the wall speed, i.e. at larger $S$, for the yield-stress fluid than for the Newtonian fluid (Fig. 1p vs. 1 k). This is because the angular velocity stays almost constant in the quasi solid-rotation zone of the yield-stress fluid, but this zone is sheared for the Newtonian fluid and the angular velocity profile decays much faster with the distance to the wall.
The images are processed to calculate the variance of the dye concentration inside the mixing region, and to measure the mixing region's area. In the variance measurements, we observe two distinct regimes of mixing (see Fig. 22). Initially, a rapid exponential mixing occurs where the dye is quickly stretched and folded until it almost fills the entire mixing region. Then a slower exponential mixing regime occurs where a minority of dye filaments that are not yet stretched to the width of the diffusion length are progressively refined through additional stretching and folding [14. From these two regimes, we can define two indices of the quality of mixing: (1) the level of mixing accomplished at the end of the first, rapid mixing regime and (2) the slope in the slower regime, i.e., the rate of mixing at long times. In the slower regime, the mixing pattern has converged to the slowest decaying advection-diffusion eigenmode [7, 15], and the decay rate is the corresponding eigenvalue.

The effect of the yield stress is observed in experiments with different fluid rheologies where the $S$ ratio is constant, but the absolute velocity changes. Fig. 2 shows results for several experiments each with an $S$ ratio of 10.33. For the same yield stress fluid, increasing the velocity increases the stress in the fluid, which leads to an increase in the amount of fluid flowing during one period. 


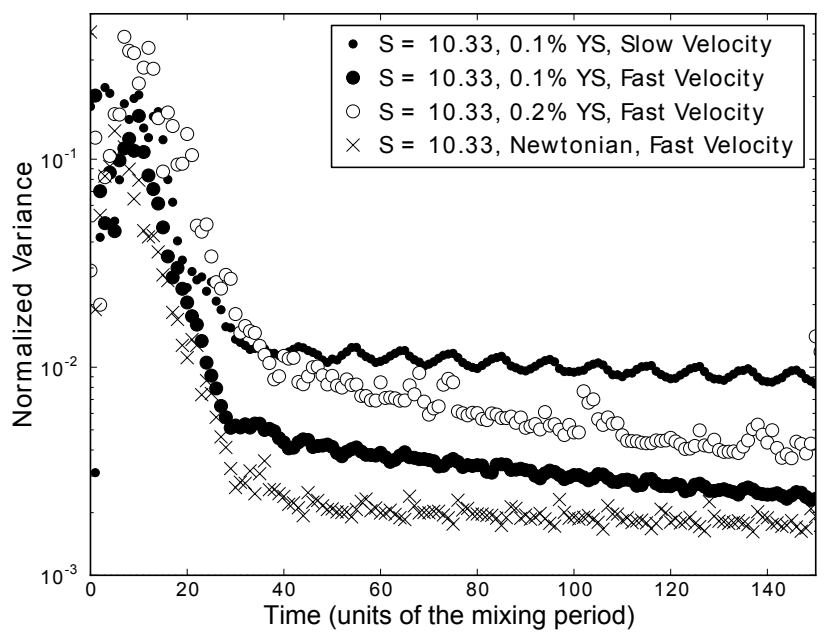

FIG. 2. Variance for different fluids mixed with a stirring ratio of $S=10.33$. The same stirring ratio can give different mixing rates based on the rheology of the fluid and the stirring velocity. Representative mixing images are seen in Fig. 1 1 -d.

Experimentally, doubling the velocity led to increased mixing during both the initial fast regime and secondary slower mixing regime. The origin of this difference is apparent on the dye patterns of Fig. 1(c) and 1(d): the contrast between the core of the mixing region and the lower peripheral part of the pattern is higher for the slow velocity. This reveals poor transport between the two regions, due to the even more intermittent stretching in the case of the slow velocity. Similarly, using the same mixing velocity but a higher yield stress value fluid shows that the higher yield stress fluid mixes slower. The Newtonian fluid mixes better than any of the yield stress fluids.

We now investigate the effect of wall speed on the mixing dynamics. It was shown in [7] that the decay rate of the variance in a Newtonian fluid and in the single injection point regime increases with increasing wall speed. However, we observe a different situation in experiments with $0.1 \%$ YS fluid, where lobes are observed at larger S-ratio. Fig. 3 shows the variance of experiments with three different wall speeds. Although at the end of the rapid mixing regime the lowest $S$ ratio has mixed the best, it is clear during the second slower mixing regime that the long-time rate of mixing increases with increasing $S$ ratio. Hence, a high long-time decay rate seems to correlate with a high level of variance when the second phase begins. This can be traced back to the size of the slow-stretching peripheral region: tiny lobes are observed for $S=4.33$, larger lobes for $S=10.33$, and a large cusp, for $S=25.83$, displays important concentration fluctuations on both sides of the injection point 7 (see Fig. 1g,d,h). This peripheral region represents the region of slowest stretching - because of intermittent flow of the yield-stress fluid - of the chaotic region, and the

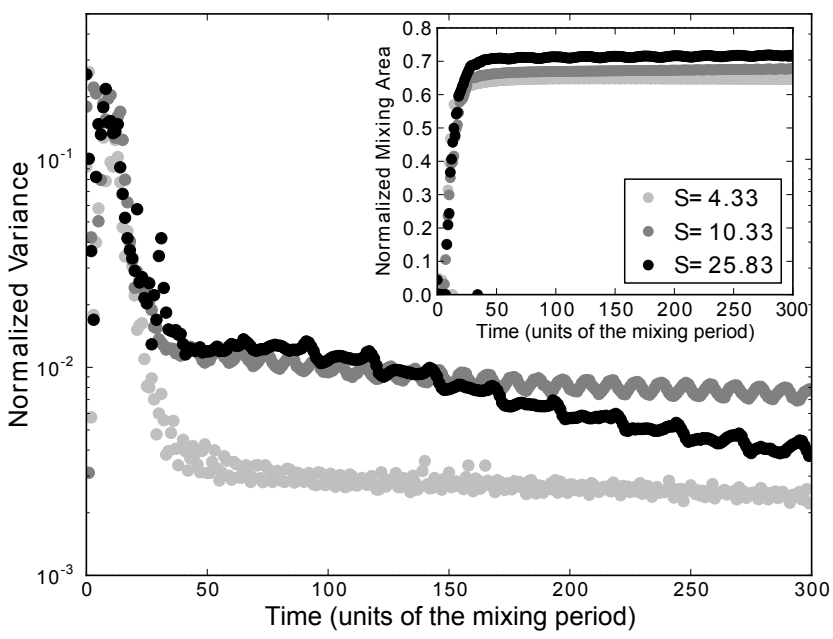

FIG. 3. Mixing rates of $0.1 \%$ YS fluid for three different vessel wall speeds, all with a stirring rod period of 6 sec. After the first mixing regime, the smaller $S$ ratio is the best mixed, but the rate of mixing is higher with larger $S$ ratios at long time. Inset: mixing area as a fraction of total vessel area for each of the $S$ ratios. Typical patterns can be seen in Fig. 1 $\mathrm{d}, \mathrm{g}, \mathrm{h}$.

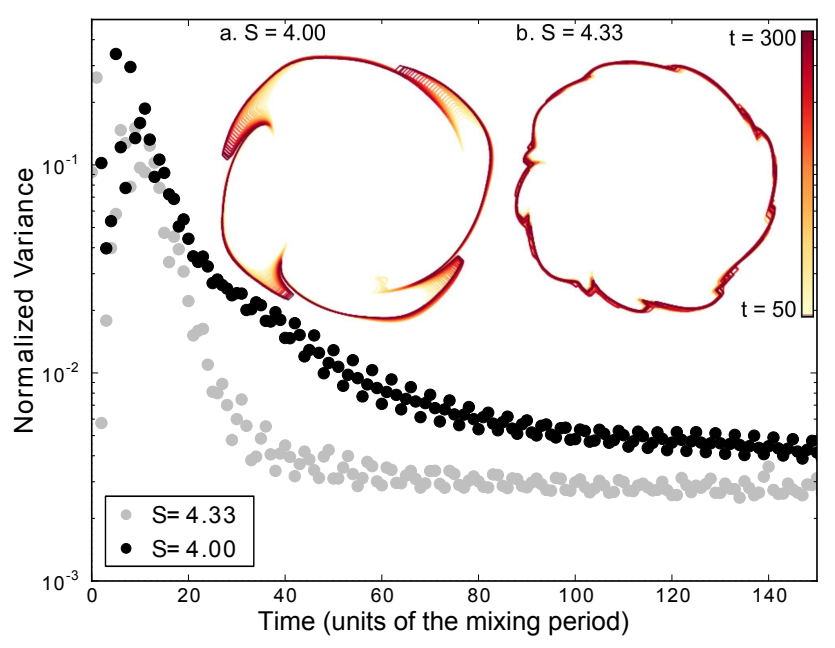

FIG. 4. (Color online) Mixing rates of $0.1 \%$ YS fluid for two different vessel wall speeds. Note the slow mixing for $S=4.00$ as compared to the $S=4.33$ case. Representative mixing images can be seen in Fig. 1; g. Inset: time evolution of the edge of the mixing area, every 4 rotation periods of the stirring rods, periods $(t) 50$ through 300 .

long-time decay is controlled by the transport of unmixed fluid from the peripheral region to the center. The larger the peripheral region, the faster the transport rate, but the higher the quantity of unmixed fluid transported to the center. The inset in Fig. 3 confirms that the mixing area increases with increasing $S$ ratio.

Important changes in mixing quality can also result 


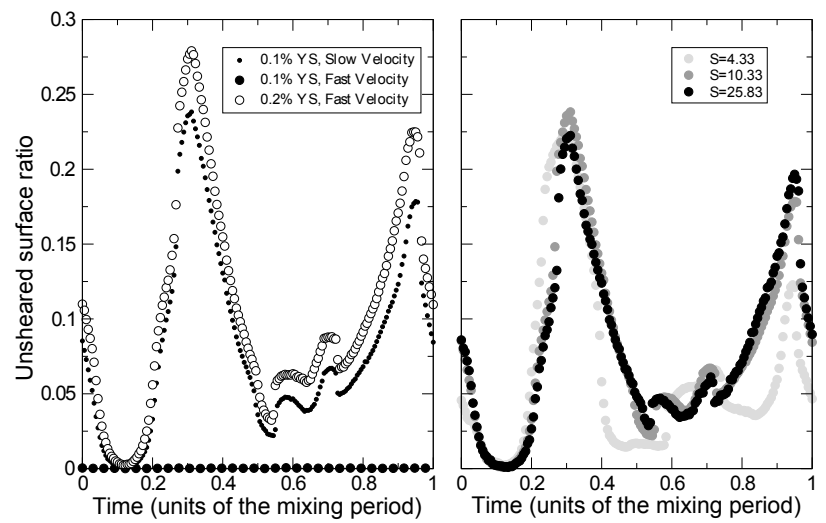

FIG. 5. Unsheared surface fraction (the local shear stress is below the yield stress) of a disk of radius $6.3 \mathrm{~cm}$ obtained in numerical simulations vs. time, during one stirring period. (a) Same conditions as Fig. 2 (different rheologies). (b) Same conditions as Fig. 3 (different wall speeds).

from a small modification of the $S$ ratio, as shown in Fig. 4. If we increase further the wall speed from an $S$ ratio of 4.33 to an $S$ ratio of 4.00 , we observe an intermediate mixing regime which decreases the efficacy of mixing in Fig. 4. This additional regime at $S=4$ comes from a resonance phenomenon: at the boundary of the mixing region, some fluid particles are entrained in solid rotation with the vessel, and are synchronized with the rod's motion because of the integer stirring ratio. As a result, the tip of the four lobes in Fig. 1: dodges repeatedly the shearing action of the rods, resulting in regions where stretching is anomalously slow. This is confirmed by the time-evolution of the mixing area boundary, delineated in Fig. 4 (inset): whereas the final shape is reached within 50 periods for $S=4.33$, in the $S=4.00$ case the final mixing area is not achieved until 150 periods, due to the slow development of lobes. In contrast, for the noninteger ration $S=4.33$, peripheral fluid particles are not synchronized with the rods and experience a succession of low and high stretchings that self-average randomly [16 over the stirring periods. Sharp changes of the mixing rate at integer stirring ratios have also been observed in numerical simulations of temperature transport [17]: for scalar transport, we propose here that this resonance is due to the repetition of slow stretching events.

We have investigated in numerical simulations how the fraction of the mixing region inside a circle of radius 6.3 $\mathrm{cm}$ and below the yield stress (shown in Fig. 5) correlates with the decay of the variance observed in experiments. The value of this non-flowing fraction fluctuates strongly during one stirring period, since the distance between the rods and the wall varies. For the fast velocity of the $0.1 \%$ YS fluid, all the fluid is sheared at all times in this region. When averaged over one period, the nonflowing fraction ranks protocols in the same order as the level of the concentration variance at the end of the fast mixing regime. Indeed, the first fast mixing regime is well described by the global transport within the mixing region. However, this sorting is no longer valid for the long-time decay rate (Fig. 5 and Fig. 22). This surprising result arises from slight Lagrangian correlations of lowstretching regions governing the long-time decay. In these chaotic flows, such correlations are hard to predict but in the resonant case (e.g. $S=4.00$ ), where correlations are enhanced. Therefore a simple link does not exist between rheological properties and long time efficiency of the mixing protocol.

The rheological properties play an important role in the efficacy of chaotic mixing. We have shown that the decreasing efficiency using chaotic advection to mix yield stress fluids compared to Newtonian fluids is due to a major mechanism: the Lagrangian correlation of intermittent flow of fluid when it is below the yield stress. Such fluid particles are found in particular at the periphery of the chaotic region, where the rotating wall entrains particles in solid rotation, except when they are sheared transiently by the passing rods. At long time, the exponential mixing rate is controlled by the transport of fluid between the peripheral region and the center. Resonances at integer stirring ratios allow some fluid particles of the chaotic region to experience repeatedly a very low stretching value, resulting in anomalously slow transport and mixing. Hence, integer ratios should be avoided for practical applications. In future work, we plan to compare numerically the Lagrangian statistics of stretching for the different protocols.

The authors acknowledge support from the ANR (project Rheomel ANR-11-JS09-015) and helpful discussions with Ph. Coussot.

[1] P. E. Arratia, J. Kukura, J. Lacombe, and F. J. Muzzio, AIChE Journal 52, 2310 (2006).

[2] H. Aref, J. Fluid Mech. 143, 1 (1984).

[3] J. M. Ottino, Rev. Fluid Mech. 22, 207 (1990).

[4] P. D. Anderson, O. S. Galaktionov, G. W. M. Peters, F. N. van de Vosse, and H. E. H. Meijer, J. Non-Newt. Fluid Mech. 93, 265 (2000).

[5] T. C. Niederkorn and J. M. Ottino, AIChE Journal 40, 1782 (1994).

[6] Y. Fan, N. Phan-Thien, and R. I. Tanner, J. Fluid Mech. 431, 65 (2001).

[7] E. Gouillart, J.-L. Thiffeault, and O. Dauchot, Phys. Rev. Lett. 104, 204502 (2010).

[8] E. Gouillart, N. Kuncio, O. Dauchot, B. Dubrulle, S. Roux, and J.-L. Thiffeault, Phys. Rev. Lett. 99, 114501 (2007).

[9] J.-L. Thiffeault, E. Gouillart, and O. Dauchot, Phys. Rev. E 84, 036313 (2011).

[10] Hershel-Bulkley fluid rheology presents a yield stress and follows the stress equation $\tau=\tau_{0}+\kappa \dot{\gamma}^{n}$ where $\tau$ is the stress, $\tau_{0}$ is the yield stress, $\kappa$ is the consistency value, $\dot{\gamma}$ 
is the shear rate and $n$ is the flow index. The measured values were $\tau_{0}=3 \mathrm{~Pa}, \kappa=4 \mathrm{~Pa} . \mathrm{s}^{n}$, and $n=0.45$ for the 0.1 w.t. $\%$ solution of Carbopol, and $\tau_{0}=18 \mathrm{~Pa}, \kappa=$ $15 \mathrm{~Pa} . \mathrm{s}^{n}$, and $n=0.4$ for the 0.2 w.t. $\%$ solution.

[11] B. D. de Besses, A. Magnin, and P. Jay, J. of Non-Newt. Fluid Mech. 115, 27 (2003).

[12] M. A. Stremler, S. D. Ross, P. Grover, and P. Kumar, Phys. Rev. Lett. 106, 114101 (2011).

[13] J. Thiffeault, M. Finn, E. Gouillart, and T. Hall, Chaos 18, 033123 (2008).
[14] A periodic variation occurs in each experiment. This periodicity comes from the period of rotation of the mixing vessel and a slight misalignment between the centerline of the mixing vessel and its rotating axis.

[15] J. Sukhatme and R. T. Pierrehumbert, Phys. Rev. E 66, 056302 (2002).

[16] E. Villermaux and J. Duplat, Phys. Rev. Lett. 91, 184501 (2003).

[17] D. Lester, M. Rudman, and G. Metcalfe, Int. J. of Heat and Mass Transfer 52, 655 (2009). 